\title{
Pediatric Inflammatory Multisystem Syndrome Associated With SARS-CoV-2: A Retrospective Cohort Study From Argentina
}

\author{
Maria Teresa Rosanova, ${ }^{1}$ Guadalupe Perez, ${ }^{1}$ Maria Martha Katsicas, ${ }^{2}$ Ana Paula Arias, ${ }^{1}$ Micela Picollo, ${ }^{2}$ \\ Marcela Palladino, ${ }^{3}$ Claudia Gonzalez, ${ }^{3}$ Natalia Veliz, ${ }^{3}$ Ana Buchovsky, ${ }^{4}$ Roberto Lede ${ }^{\mathbf{5}}$, Rosa Bologna ${ }^{1}$ \\ From Departments of ${ }^{1}$ Infectious Diseases Hospital Juan P Garrahan Combate de los Pozos 1881, ${ }^{2}$ Rheumatology, Hospital Juan P \\ Garrahan Combate de los Pozos 1881, ${ }^{3}$ Clinics, 75, Hospital Juan P Garrahan Combate de los Pozos 1881, ${ }^{4}$ Serology, Hospital \\ Juan P Garrahan Combate de los Pozos 1881, ${ }^{5}$ Universidad Abierta Interamericana (UAI), Avenida San Juan 961, Buenos Aires; \\ Argentina.
}

Correspondence to: Dr Maria Teresa Rosanova, Chief of Clinics, Department of Infectious Diseases, Hospital Juan P Garrahan, Combate de los Pozos 1881 Buenos Aires, Argentina.maritesolcito@gmail.com Received: December 16, 2020; Initial review: January 8, 2021; Accepted: April 16, 2021.
Objective: To evaluate the differential characteristics of SARS-COV-2 associated inflammatory multisystem syndrome (MIS-C) in children. Methods: A retrospective cohort study was conducted. The definition of MIS- $\mathrm{C}$ was based on WHO criteria. Temporally related COVID19 patients were included as controls. Results: 25 patients with MIS-C and 75 controls were included. Multivariate multiple logistic regression model of variables that showed to be significant in univariate analysis revealed that age $\geq 2$ years (OR 24.7; 95\% Cl $1.03-592.4$; $P=0.048$ ), Iymphopenia (OR $9.03,95 \% \mathrm{Cl} 2.05-39.7 ; P=0.004$ ), and platelet count $<150 \times 10^{9}$ / L (OR $11.7 ; 95 \% \mathrm{Cl} 1.88-75.22 ; P=0.009)$ were significantly associated with MIS-C. Presence of underlying disease seemed to reduce the risk of MIS-C (OR 0.06; 95\% $\mathrm{Cl} 0.01-0.3$ ). Conclusion: MIS-C was more common in patients older than 2 years and in those with lymphopenia or thrombocytopenia. Underlying disease appears to reduce the risk of MIS-C.

Keywords:Co-morbidity, Outcome, Lymphopenia, Thrombocytopenia.

Publised online: April 17, 2021; PII: S097475591600313

$\mathrm{M}$ ultisystem inflammatory syndrome (MIS-C), in association with severe acute respiratory syndrome coronavirus 2 (SARS-CoV-2) infection, shares some clinical features with Kawasaki disease (KD), toxic shock syndrome (TSS), macrophage activation syndrome, and other inflammatory processes as in the so-called cytokine storm [1-5]. MIS-C is characterized by persistent fever, abdominal pain, vomiting, diarrhea, as well as mucocutaneous, cardiovascular, hematological, musculoskeletal, and neurological manifestations, among others [611]. After identification of MIS-C cases in Argentinean children with COVID-19, we conducted this study to evaluate the possible risk factors associated with MIS-C to allow clinicians to categorize patients who may require closer monitoring and interdisciplinary management.

\section{METHODS}

A retrospective cohort study was conducted at a tertiary pediatric referral center. Patients were identified from the electronic database of pediatric patients with confirmed COVID-19 seen between 19 April, and 31 October, 2020, at the Department of infectious diseases. This study was approved by the institutional research board.
Children with a diagnosis of MIS-C, as established by the World Health Organization (WHO) [12], were included in the study and defined as cases. For each case, three consecutive children with a positive reverse transcriptasepolymerase chain reaction (RT-PCR) for SARS-CoV-2 and no clinical or laboratory findings suspicions of MIS-C were selected as controls. Thrombocytopenia was defined as a platelet count $<150 \times 10^{9} / \mathrm{L}$ and lymphopenia as a lymphocyte count $<1 \times 10^{9} / \mathrm{L}$ on admission. C-reactive protein (CRP) $<5$ mg/L, B-type natriuretic peptide (BNP) $<104$ pg/ $\mathrm{mL}$, troponin $<19 \mathrm{ng} / \mathrm{L}$, and ferritin $<200 \mathrm{ng} / \mathrm{mL}$ were considered to be within normal range. Cardio-vascular involvement was identified in the presence of any of the following: vasopressor requirement, an echo-cardiogram showing an abnormal ejection fraction, pericarditis or pericardial effusion, or elevated troponin or BNP levels. For both cases and controls, exclusion criteria were previous treatment with convalescent plasma or steroids, presence of any viral or bacterial co-infections, and outpatient status.

We analyzed epidemiological data (age, sex, comorbidities, overcrowding defined as more than 4 people living in one room, and living in informal settlements); 
virological data (RT-PCR of nasopharyngeal secretions and/or positive serology on admission); and clinical data (fever, respiratory distress, abdominal pain, diarrhea, vomiting, myalgia, dysgeusia, anosmia, conjunctivitis, rash, shock, intensive care unit (ICU) admission, mechanical ventilation requirement, oxygen therapy, or inotropic and vasopressors, and the length of hospital stay).

Laboratory tests on admission included leukocyte, lymphocyte, and platelet counts. In patients with suspected MIS-C, coagulogram, fibrinogen, ferritin, CRP, procalcitonin (PCT), liver and kidney function, lactate dehydrogenase (LDH), BNP, and troponin were analyzed (when available). Echocardiogram and radiographs were performed on admission and repeated depending on clinical features. Lung computed tomography (CT) scan and abdominal ultrasound were performed according to symptoms. Blood and urine cultures, PCR of nasopharyngeal swabs for influenza virus, respiratory syncytial virus, adenovirus, metapneumovirus, rhinovirus, and coronaviruses and serology for HIV, VDRL, HCV, HBV, $\mathrm{CMV}$ and $\mathrm{EBV}$ were also performed. Intravenous immunoglobulin (IVIG) and/or steroid use was documented. Outcome was defined as discharge or death.

Statistical analysis: Univariate analysis was performed to compare cases and controls. Odds ratio (OR) with a 95\% CI was used for dichotomous variables. Chi-square or rank-sum test were used. Predictive factors for MIS-C were identified using a multiple logistic regression model including variables that were significant in univariate analysis. STATA 16 was used for statistical analysis. A value of $P<0.05$ was considered as significant.

\section{RESULTS}

Of the 533 children aged $<18$ years with COVID-19, 25 (4.7\%) met the diagnostic criteria for MIS-C. In addition, 75 patients were included as controls. Median age of cases was 104 months (IQR 61-126) vs 78 months (IQR 18-139) in the control group. Underlying diseases were more commonly observed in the control group $(n=52$; $69 \%)$ than in MIS-C patients $(n=7 ; 28 \%)$ Underlying diseases were cancer (18 controls; 24\%), solid organ trans-plantation (18 controls; $24 \%$ ), genetic disorders (4 controls; $5 \%$ ), neurological disorders (4 controls; $5 \%$, and 3 cases; $12 \%$ ), congenital disorders ( 3 controls; $4 \%$ ), obesity ( 3 controls; $4 \%$ ), recurrent wheezing ( 3 cases; $12 \%)$, chronic renal failure ( 1 case; $4 \%$ ), and others (15 controls; 20\%).

Clinical manifestations of MIS-C patients and controls are shown in Table I. Nine MIS-C patients $(36 \%)$ vs one control (1.3\%) required intensive care unit
Table I Characteristics of Children With SARS-CoV-2 Infection With and Without MIS-C

\begin{tabular}{|c|c|c|c|}
\hline Variable & $\begin{array}{l}M I S-C \\
n=25 \\
\end{array}$ & $\begin{array}{l}\text { Controls } \\
n=75\end{array}$ & OR (CI 95\%) \\
\hline Male sex & $9(36)$ & $36(48)$ & $\begin{array}{r}0.61 \\
(0.21-1.69)\end{array}$ \\
\hline Age, $\mathrm{mo}^{a}$ & $\begin{array}{l}104 \\
(61-126)\end{array}$ & $\begin{array}{l}78 \\
(18-139)\end{array}$ & ) \\
\hline Age $\geq 2 y$ & $24(96)$ & $54(72)$ & $\begin{array}{r}9.33 \\
(1.19-73.4)\end{array}$ \\
\hline $\begin{array}{l}\text { Living in a popular } \\
\text { neighborhood }\end{array}$ & $8(32)$ & $7(9)$ & $\begin{array}{r}4.57 \\
(1.23-16.8)\end{array}$ \\
\hline Asymptomatic & 0 & $20(27)$ & - \\
\hline Fever & $25(100)$ & $29(39)$ & - \\
\hline Abdominal pain & $9(36)$ & $10(13)$ & $2.68(0.8-8.2)$ \\
\hline Diarrhea & $14(56)$ & $9(12)$ & $9.33(2.9-30.5)$ \\
\hline Vomiting & $12(48)$ & $13(17)$ & $4.40(1.4-13)$ \\
\hline Skin involvement & $16(64)$ & $3(4)$ & $\begin{array}{r}42.66 \\
(9.1-254.8)\end{array}$ \\
\hline Conjunctival injection & $9(36)$ & 0 & - \\
\hline Septic shock & $8(32)$ & 0 & - \\
\hline $\begin{array}{l}\text { Upper respiratory } \\
\text { tract infection }^{b}\end{array}$ & $5(20)$ & $18(24)$ & $\begin{array}{r}0.79 \\
(0.2-2.6)\end{array}$ \\
\hline Odynophagia & $4(16)$ & $8(11)$ & $1.59(0.3-6.7)$ \\
\hline Tachypnea $^{c}$ & $3(12)$ & $2(3)$ & $4.97(0.5-61)$ \\
\hline Headache & $2(8)$ & $4(5)$ & $1.54(0.1-11.5)$ \\
\hline Myalgia & $3(12)$ & $1(1) \quad 10$ & $0.09(0.7-536.6)$ \\
\hline Anosmia & 0 & $1(1)$ & - \\
\hline
\end{tabular}

Values in no. (\%) or ${ }^{a}$ median (IQR). ${ }^{b}$ Upper respiratory tract infection: cough, loss of appetite, sore throat, or nasal congestion, nasal stuffiness, rhinorrhea, anosmia; ${ }^{\circ}$ Tachypnea (breaths $/ \mathrm{min}$ ): $\leq 2 \mathrm{mo}:>60 ; 2-11 \mathrm{mo}$ : $>50 ; 1-5 y:>40 ;>5 y:>20$. MIS-C: multi-system inflammatory syndrome associated with SARS-CoV-2 in children; PCR: polymerase chain reaction.

(ICU) admission. Eight MIS-C patients (32\%) had cardiac abnormalities consisting of myocarditis $(n=3)$, pericarditis $(n=1)$, left ventricular dysfunction $(n=3)$ and coronary dilatation $(n=1)$. Lymphopenia and thrombocytopenia were more common in MIS-C patients than controls. Median (IQR) CRP was 139 (122-248) mg/L, BNP $1116(183-4857) \mathrm{pg} / \mathrm{mL}$, troponin $2.5(<1.5-79) \mathrm{ng} /$ $\mathrm{L}$, and ferritin 339 (191-611) ng/mL in MIS-C patients. At onset, PCR for SARS-Co-V2 was positive in 15 MIS-C patients $(60 \%)$ and in all of those in the control group. IgM antibodies were positive in seven MIS-C patients $(28 \%)$ and six controls ( $8 \%$ ), while IgG antibodies were positive in 24 MIS-C patients (96\%) and 22 controls (29\%). In one MIS-C patient with a history of close contact with COVID-19 one month previously, both the PCR and antibody test were negative. All MIS-C patients 
received IVIG, associated with steroids in $15(60 \%)$. Median (IQR) length of hospital stay was 10 days (9-12) in cases vs 8 (4-11) days in controls ( $P=0.006)$ (Table II).

Multivariate logistic regression analysis revealed that age $>2$ years (OR 24.71; 95\%CI,1.03-592.42), lymphopenia (OR 9.03; 95\% CI, 2.05-39.70), and thrombocytopenia (OR 11.73; 95\% CI, 1.88-75.22) at diagnosis were significantly associated with MIS-C. Presence of underlying diseases (OR 0.06; 95\%CI, 0.01-0.30) seems to reduce the probability of developing MIS-C.

\section{DISCUSSION}

In our series all MIS-C patients were older than 2 years, which is in agreement with previous finding that MIS-C does not affect infants younger than 1 year [1-8]. Potential age-related variability among MIS-C patients may result from differences in SARS-CoV-2 infection due to the likelihood of exposure or to differential nasal expression of angiotensin-converting enzyme 2 , the entry receptor for SARS-CoV-2 [2-6]. Inequalities in access to health care, genetics-related risks, or overcrowding might be lines for future research. In our study, living with a person with COVID-19 and overcrowded living conditions were significantly associated with MIS-C in univariate analysis.

Several studies evaluating patients with MIS-C have found high rates of comorbidities $[8,13,14]$. In our series comorbidities were less frequently observed in MIS-C patients than in controls. A recent meta-analysis concluded that cancer patients undergoing treatment were not at a higher risk of developing more severe COVID-19 disease; however, the role of comorbidities in patients with COVID-19 needs further exploration [15]. Most MIS-C patients had a previous or concurrent laboratoryconfirmed SARS-CoV-2 infection, supporting the hypothesis that MIS-C is an immune-mediated postinfectious synd-rome related to SARS-CoV-2 infection [6-11]. In our series, gastrointestinal symptoms were more frequent in MIS-C patients than in controls. Fever was observed in all MIS-C patients but not in all controls [8]. Similar to other series, shock and acute heart failure were more frequent in MIS-C patients than in controls [13].

MIS-C is associated with heart complications and inflammatory disorders triggered by SARS-CoV-2 with features similar to KD suggesting that this virus might be acting as an immunological trigger causing similar immune- mediated injury to the heart and coronary arteries comparable to KD [13]. Cardiovascular involvement was common in our MIS-C patients but not seen in any of the controls.

Low lymphocyte count, associated with poor outcome, was more common in MIS-C patients than in controls [14]. Levels of acute-phase reactants (CRP and PCT), may be high in patients with MIS-C [5,8]. Similar to other studies, all MIS-C patients received IVIG and combination with steroids in some of them [8]. As in previous studies on COVID-19 in children, ICU admission was more frequent in MIS-C patients [7-8], but no deaths were reported.

In conclusion SARS-CoV-2 associated MIS-C was more common in children older than 2 years and in those with lymphopenia or thrombocytopenia. The presence of underlying diseases seems to decrease the likehood to develop MIS-C; however, further studies are needed to confirm this observation and rule out that it was an incidental association in this study.

Ethics clearence: Hospital Juan P Garrahan; No. 1294, dated October 11, 2020.

Table II Laboratory Characteristics of Children With SARS-CoV-2 Infection With and Without MIS-C at Hospital Admission

\begin{tabular}{lllr}
\hline Variable & MIS-C, $n=25(\%)$ & Controls,$n=75(\%)$ & OR $($ CI 95\%) \\
\hline White cells count $^{a}$ & $10650(6800-13680)$ & $6840(4350-10830)$ & - \\
Lymphopenia & $15(60)$ & $14(19)$ & $4.8(1.77-13.1)$ \\
Platelets count/L ${ }^{a}$ & $197000(137000-246000)$ & $294000(210000-375000)$ & - \\
Thrombocytopenia $_{\text {C-reactive protein, } \mathrm{mg} / \mathrm{L}^{a}}$ & $8(32)$ & $4(5)$ & $8.3(2.24-31)$ \\
Abnormal liver enzymes & $139.9(122-248.5)$ & $2.94(0.77-22.8)$ & - \\
SARS-CoV-2 PCR & $3(12)$ & $5(7)$ & $1.9(0.3-10.7)$ \\
IgM SARS-CoV-2 & $15(60)$ & $75(100)$ & - \\
IgG SARS-CoV-2 & $7(28)$ & $6(8)$ & $4.47(1.1-18.0)$ \\
\hline
\end{tabular}

Values in no. (\%) or ${ }^{a}$ median (IQR). Lymphopenia-lymphocyte count $<1000 / \mathrm{mm}^{3}$ on admission; Thrombocytopenia: platelet count $<150000 / \mathrm{mm}^{3}$. MIS-C-multisystem inflammatory syndrome associated with SARS-CoV-2 in children; PCR -polymerase chain reaction. 


\section{WHAT THIS STUDY ADDS?}

- In Argentina, MIS-C was more common in children older than two years and those with lymphopenia or thrombocytopenia.

- Presence of underlying diseases seems to decrease the likelihood to develop MIS-C.

Contributors: MTR: conceptualized the study design; analyzed and interpreted the results, and wrote the manuscript; GP, MMK, AAP, MP, CG, NB, AB: recruited patients, collected demographic and clinical data analyzed and interpreted the results; RL,RB: conceptualized the study design- analyzed and interpreted the results, and commented on and revised the manuscript. All authors approved the final version of manuscript, and are accountable for all aspects related to the study.

Funding: None; Competing interest: None stated.

\section{REFERENCES}

1. Jones VG, Mills M, Suarez D, et al. COVID-19 and Kawasaki disease: Novel virus and novel case. Hosp Pediatr. 2020;10:537-40.

2. Zimmermann $P$, Curtis N. Coronavirus infections in children including COVID-19: An overview of the epidemiology, clinical features, diagnosis, treatment and prevention options in children. Pediatr Infect Dis J. 2020;39:355-68.

3. CDC. Multisystem inflammatory syndrome in children (MIS-C) associated with coronavirus disease 2019 (COVID-19). Accessed December 1, 2020. Available from: https://www.cdc.Gov/coronavirus/2019-ncov/daily-lifecoping/children/mis-c.html

4. Royal College of Paediatrics and Child Health. Guidance: Paediatric multisystem inflammatory syndrome temporally associated with COVID-19. Accessed September 25, 2020. Available from: https://www.rcpch.ac.uk/sites/default/files/ 2020-05/COVID19-Paediatric-multisystem-inflammatory syndrome-20200501.pdf.

5. Henderson LA, Canna SW, Friedman KG, et al. American College of Rheumatology Clinical Guidance for Multisystem Inflammatory Syndrome in Children Associated With SARS-CoV2 and Hyperinflammation in Pediatric COVID19: Version 1. Arthritis Rheumatol. 2020;72:1791-805.
6. Shobhavat L, Solomon R, Rao S, et al. Multisystem inflammatory syndrome in children: Clinical features and management-intensive care experience from a pediatric public hospital in Western India. Indian J Crit Care Med. 2020;24:1089-1094.

7. García-Salido A, de Carlos Vicente JC, Belda Hofheinz S, et al. Severe manifestations of SARS-CoV-2 in children and adolescents: From COVID-19 pneumonia to multisystem inflammatory syndrome: A multicentre study in pediatric intensive care units in Spain. Crit Care. 2020; 24:1-13.

8. Feldstein LR, Rose EB, Horwitz SM, et al. Multisystem inflammatory syndrome in US children and adolescents. $\mathrm{N}$ Engl J Med. 2020;383:334-46.

9. Jiang L, Tang K, Levin M, et al. COVID-19 and multisystem inflammatory syndrome in children and adolescents. Lancet Infect Dis. 2020; 20:e276-e288.

10. Radia T, Williams N, Agrawal P, et al. Multi-system inflammatory syndrome in children and adolescents (MISC): A systematic review of clinical features and presentation. Paediatr Respir Rev. 2020:S1526-1542.

11. WHO. Multisystem inflammatory syndrome in children and adolescents with COVID-19. Accessed November 25, 2020. Available from: https://www.who.int/publications/i/ item/ multisystem-inflammatory-syndrome-in-childrenand-adolescents-with-covid-19

12. Rodriguez-Gonzalez M, Castellano-Martinez A, CascalesPoyatos HM, et al. Cardiovascular impact of COVID-19 with a focus on children: A systematic review. World J Clin Cases. 2020; 8:5250-283.

13. Huang I, Pranata R. Lymphopenia in severe coronavirus disease - 20192738 (COVID 19): Systematic review and meta-analysis. J Intensive Care. 2020;8:1-10.

14. Yekedüz E, Utkan G, Ürün Y. A systematic review and meta-analysis: The effect of active cancer treatment on severity of COVID-19. Eur J Cancer. 2020;141:92-104. 